\title{
Time-Delay Effects on Synchronization of Coupled Slow-Fast Systems
}

\author{
Yuanguang Zheng*, Jianjian Zeng \\ School of Mathematics and Information Science, Nanchang Hangkong University, Nanchang, China \\ Email: *zhengyg2718@163.com
}

How to cite this paper: Zheng, Y.G. and Zeng, J.J. (2021) Time-Delay Effects on Synchronization of Coupled Slow-Fast Systems. Journal of Applied Mathematics and Physics, 9, 635-647. https://doi.org/10.4236/jamp.2021.94046

Received: March 10, 2021

Accepted: April 17, 2021

Published: April 20, 2021

Copyright $\odot 2021$ by author(s) and Scientific Research Publishing Inc. This work is licensed under the Creative Commons Attribution International License (CC BY 4.0).

http://creativecommons.org/licenses/by/4.0/

(c) (i) Open Access

\begin{abstract}
Time-delay effects on synchronization features of delay-coupled slow-fast van der Pol systems are investigated in the present paper. The synchronization mechanism of "slow-manifold adjustment" is firstly described on the basis of geometric singular perturbation theory. Then, the impact of time delay on the structure of the slow manifold of synchronized system is obtained by using the method of stability switch, and thus, time-delay effects on synchronization features are stated. It is shown the time delay cannot qualitatively affect the synchronization mechanism, however, it can result in the drift of the optimal coupling strength.
\end{abstract}

\section{Keywords}

Synchronization, Slow-Fast System, Time-Delay, Geometric Singular Perturbation Theory

\section{Introduction}

Real system is often with two kinds of different dynamical variables, evolving on very different timescales [1] [2], this system is called slow-fast system, and the rapid evolving and slowly evolving variables are called fast and slow variables respectively. van der Pol system with one slow variable and one fast variable is one of the typical slow-fast system [3]. Though simple enough for analytical investigation, that system can exhibit various dynamical behaviors due to the interaction of the fast and slow variables, such as relaxation oscillation, nonsmooth limit cycle, French duck solution, etc. [3] [4] [5] [6]. Relaxation oscillation is universal in real systems, such as electrical circuits [3], ecosystems [7], chemical systems [8], and biological neural systems [9] [10] [11]. Relaxation oscillation is characterized by the rapid movement process alternating with the slow move- 
ment process, which is different from harmonic oscillation [12]. Geometric singular perturbation theory [13] [14] [15] [16] is an efficient tool to describe the generation of relaxation oscillation. This theory defines a slow manifold of slow-fast system, which is an approximation of the invariant manifold, and it indicates the evolution of solution trajectory is governed by the structure of the slow manifold including the shape, stability and bifurcation points of the slow manifold, and the solution trajectory will be attracted by stable slow manifold and repelled by unstable slow manifold.

Coupled systems will adjust their behaviors with each other, such that there are some relations between their dynamical behaviors, this phenomenon is called synchronization. Since synchronization was first reported in the study of two coupled pendulums [17], this phenomenon has got much attention and has been intensively studied in literature. Studies indicate there are various kinds of synchronization, such as phase synchronization, complete synchronization, anti-synchronization, generalized synchronization, lag synchronization, and so on [18] [19] [20]. Complete synchronization is one of the strongest synchronization, which means all the coupled systems do the same things at the same time. A striking finding is that complete synchronization can occur in coupled chaotic systems [21] [22], and this phenomenon has found wide applications in engineering [19]. To describe the stability of synchronization manifold, condition Lyapunov exponent is introduced in [23], and the synchronization manifold is stable if the largest condition Lyapunov exponent is negative, and thus, the coupled systems get synchronization. To study network-structure effects on synchronization, an universal master stability function was introduced by separating the synchronization manifold direction from other transverse directions [24].

Coupled slow-fast systems have different synchronization features to that of the coupled systems with uniform timescale. Coupled relaxation oscillators with heaviside coupling can get synchronization through the mechanism of "fast threshold modulation" [25], which is different from the phase-pulling mechanism of coupled harmonic oscillators. For weakly connected relaxation oscillators, the phase equation was derived in [26], and the mechanism of "fast threshold modulation" is conformed. The synchronization rate of coupled relaxation oscillators in one-dimensional chains with heaviside step function interaction was numerically calculated in [27], and it is shown chain-coupled relaxation oscillators can get synchronization much more rapidly than that of coupled harmonic oscillators. The chains of relaxation-type neural oscillators with local excitatory coupling is studied by using the phase reduction and fast threshold modulation theories [28], it is shown the chains undergo the transition from waves to synchronization when the system approaches the relaxation limit. Diffusive coupling relaxation oscillators in chain can exhibit a variety of complex spatial patterns, and the basins of attraction for various patterns were obtained in [29].

Time delay should be considered in coupled systems due to finite information transmission and processing speed [30] [31] [32] [33] [34]. Studies indicate time 
delay has significant effect on characteristic of synchronization. Time-delay can lead to amplitude death of two coupled limit cycle oscillators even if they have the same frequency, this is in sharp contrast to the situation without time-delay, where the amplitude death can only happened when their frequency is sufficiently disparate [30]. Cluster synchronization in nearest-neighbor delay-coupled limit-cycle oscillators was considered in [31], it is shown time delay has an important effect on the stability of various cluster states, and the mean frequency is decreasing with increase of the time-delay. Time-delay is also included in the synchronized system which describes the dynamics of synchronized state on the synchronization manifold, thus, time-delay can change the dynamics of synchronized state, and lead to complex synchronized dynamics [35] [36]. Delay-induced various transitions of synchronization are observed [37] [38]. Though time delay frequently induces complex synchronized dynamics and synchronization transitions in coupled systems, under certain conditions, time-delay can be a positive factor for synchronization [39]. Based on the plot of maximum transverse Lyapunov exponents obtained in [32], it is shown the complete synchronization occurs with very low coupling strength when connection delay is involved.

Synchronization of delay-coupled slow-fast systems has also been studied in literature [40] [41], an important finding is that the delay-coupled slow-fast systems can get synchronization in large range of connection delay, which is helpful to understand the real Neural synchrony with long connection delay [40] [41]. However, to the best of our knowledge, time-delay effect on synchronization features of delay-coupled slow-fast systems is still not fully clarified. This paper is devoted to further investigate time-delay effect on synchronization features of delay-coupled slow-fast van der Pol systems on behalf of the method of stability switch [42] and Geometric singular perturbation theory [13] [14] [15] [16]. A synchronization mechanism of "slow-manifold adjustment" is described in next section. In Section 3, time-delay influence on the structure of the slow manifold of synchronized system is firstly obtained, and then, time-delay effect on the synchronization features of coupled slow-fast van der Pol systems is stated. Some conclusions are given in the last section.

\section{Synchronization of Coupled Slow-Fast van der Pol Systems}

Two identical delay-coupled slow-fast van der Pol systems are described as

$$
\left\{\begin{array}{l}
\dot{x}_{i}=-y_{i}+x_{i}-\frac{1}{3} x_{i}^{3}+I_{1}\left(x_{j}(t-\tau)-x_{i}\right) \\
\dot{y}_{i}=\varepsilon x_{i} .
\end{array}\right.
$$

where $i=1,2$ and $j=2,1$, a dot represents derivative with respect to time $t$, $0<\varepsilon \ll 1$ such that $x_{i}$ is the fast variable and $y_{i}$ is the slow variable, $I_{1}\left(x_{j}-x_{i}\right)=c\left(x_{j}-x_{i}\right)$ is the linearly coupled term with coupling strength $c \geq 0$, and $\tau=O(1)$ represents the connection delay. 
To study time-delay effect on synchronization features of coupled systems (1), the synchronization mechanism of coupled slow-fast van der Pol systems without connection delay should be clarified firstly. Without connection delay, Equation (1) read

$$
\left\{\begin{array}{l}
\dot{x}_{i}=-y_{i}+x_{i}-\frac{1}{3} x_{i}^{3}+I_{1}\left(x_{j}-x_{i}\right) \\
\dot{y}_{i}=\varepsilon x_{i} .
\end{array}\right.
$$

In the synchronization manifold of coupled systems (2), the dynamics of synchronized state $\left(x_{1}, y_{1}\right)=\left(x_{2}, y_{2}\right)=(x, y)$ is described by

$$
\left\{\begin{array}{l}
\dot{x}=-y+x-\frac{1}{3} x^{3} \\
\dot{y}=\varepsilon x
\end{array}\right.
$$

Equation (3) is actually the single slow-fast van der Pol system with one slow and one fast variables. Let $\varepsilon \rightarrow 0$ in Equation (3), one has the fast subsystem

$$
\dot{x}=-y+x-\frac{1}{3} x^{3}
$$

where $y$ is taken as a system parameter, thus, the fast subsystem governs the fast variable $x$ only. Geometric singular perturbation theory defines the slow manifold of Equation (3) as the set of equilibrium points of the fast subsystem

$$
M_{1}=M_{11} \cup M_{12} \cup M_{13}=\left\{(x, y) \mid-y+x-\frac{1}{3} x^{3}=0\right\} \text {. }
$$

The structure of slow manifold, including the stability and bifurcation points, can be determined through the stability analysis of the fast subsystem, as shown in Figure 1(a). According to geometric singular perturbation theory, the dynamics of Equation (3) is governed by the structure of slow manifold $M_{1}$, and the solution trajectory is attracted by the stable slow manifold and repelled by the unstable slow manifold, thus, the synchronized state of Equation (3) undergoes relaxation oscillation, as shown in Figure 1(a) and Figure 1(b).

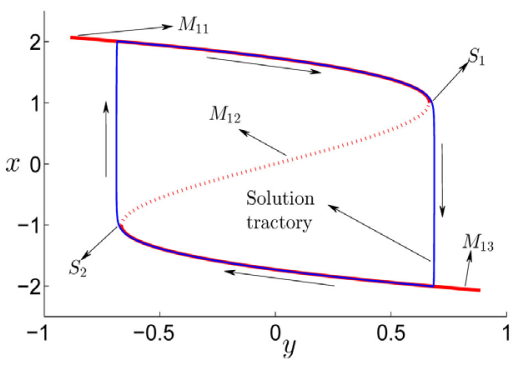

(a)

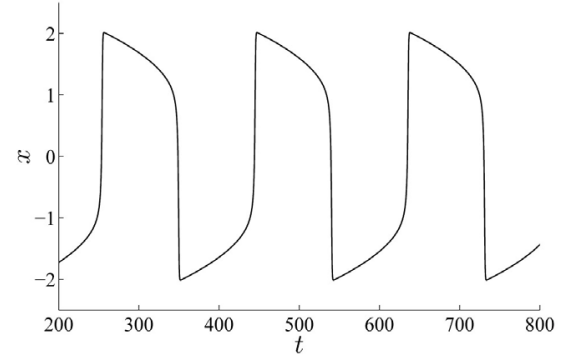

(b)

Figure 1. The structure of the slow manifold $M_{1}=M_{11} \cup M_{12} \cup M_{13}$ of Equation (3), where $M_{12}$ is unstable, $M_{11}$ and $M_{13}$ are stable, $S_{1}$ and $S_{2}$ are the saddle-note bifurcation points, and the solution trajectory is attracted by the stable slow manifold and repelled by the unstable slow manifold, thus, Equation (3) undergoes relaxation oscillation. (a) Slow manifold $M_{1}=M_{11} \cup M_{12} \cup M_{13}$ and solution trajectory; (b) Time series. 
To describe the synchronization mechanism of Equations (2) analytically, simplify the coupling term as follows

$$
I_{1}(w)=c w \Rightarrow I_{2}(w)=\left\{\begin{array}{ll}
c w & w>\delta \\
0 & |w| \leq \delta \\
c w & w<-\delta
\end{array} \Rightarrow I_{3}(w)= \begin{cases}c & w>\delta \\
0 & |w| \leq \delta \\
-c & w<-\delta\end{cases}\right.
$$

where $w=x_{j}-x_{i} \quad(i \neq j)$ and $\delta$ is a small positive constant, and this process of simplification is illustrated in Figure 2.

With the simplified coupling $I_{3}(w)$, Equations (2) read

$$
\left\{\begin{array}{l}
\dot{x}_{1}=-y_{1}+x_{1}-\frac{1}{3} x_{1}^{3}+I_{3}\left(x_{2}-x_{1}\right) \\
\dot{y}_{1}=\varepsilon x_{1}
\end{array}\right.
$$

and

$$
\left\{\begin{array}{l}
\dot{x}_{2}=-y_{2}+x_{2}-\frac{1}{3} x_{2}^{3}+I_{3}\left(x_{1}-x_{2}\right) \\
\dot{y}_{2}=\varepsilon x_{2} .
\end{array}\right.
$$

Though the coupling term of Equations (4) - (5) is the simplified version of that of Equations (2), the synchronization mechanism of Equations (4) - (5) is qualitatively the same as that of Equations (2), which will be illustrated in following discussion, and will also be confirmed by numerical results.

To describe the synchronization features of Equations (4) - (5), the phase and phase difference for relaxation oscillation is firstly introduced. As shown in Figure 3, let $T$ denotes the period of the relaxation oscillation, and $O\left(\frac{2}{3}, 1\right)$ denotes the point with zero phase, and $T_{P_{i} \rightarrow P_{j}}$ denotes the time required for point $P_{i}$ evolving to point $P_{j}$. Regard anti-clockwise direction as the forward direction, and the phase difference from point $P_{i}$ to point $P_{j}$, denoted with $T_{P_{i} P_{j}}$, is defined as $T_{P_{i} P_{j}}=T_{P_{i} \rightarrow P_{j}}$ if $T_{P_{i} \rightarrow P_{j}} \leq \frac{1}{2} T$ or $T_{P_{i} P_{j}}=-T_{P_{j} \rightarrow P_{i}}$ if $T_{P_{i} \rightarrow P_{j}}>\frac{1}{2} T$, and the phase of point $P_{i}$ is defined as the phase difference from point $P_{i}^{2}$ to point $O$, denoted as $T_{P_{i} O}$. And one has $T_{R_{1} P_{3}}=T_{P_{1} \rightarrow P_{3}}$, $T_{P_{3} P_{1}}=-T_{P_{1} \rightarrow P_{3}}=-T_{P_{1} P_{3}}, \quad T_{P_{1} P_{2}}=T_{P_{1} O}-T_{P_{2} O} \approx \int_{x_{2}}^{x_{1}}\left(\frac{1}{x}-x\right) \mathrm{d} x \approx \frac{\left|y_{1}-y_{2}\right|}{\left|x_{1}+x_{2}\right| / 2}$. Furthermore, one has $T_{P_{1} P_{2}}>T_{P_{3} P_{4}}$ due to $\left|x_{1}+x_{2}\right|<\left|x_{3}+x_{4}\right|$, and let $l=\frac{T_{P_{3} P_{4}}}{T_{P_{1} P_{2}}}<1$ denote the contraction ratio.

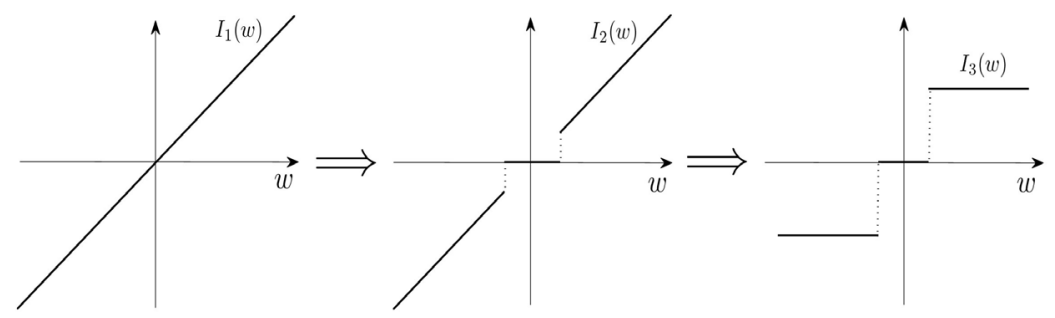

Figure 2. The simplification of the coupling terms in Equation (2). 


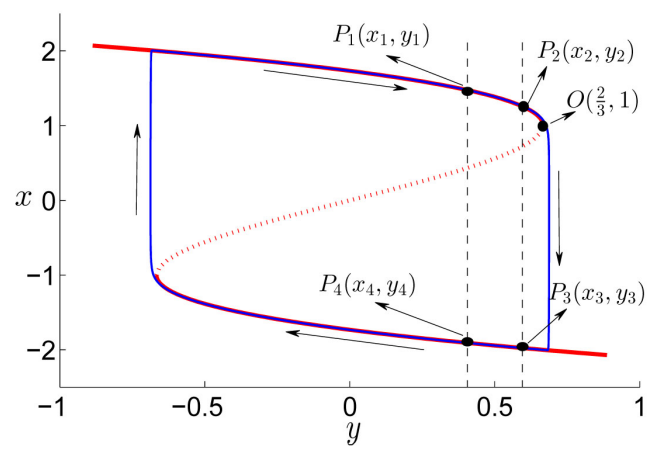

Figure 3. The phase and phase difference for relaxation oscillation of Equation (3).

Denote the initial points of Equations (4) and (5) with $A_{10}\left(x_{10}, y_{10}\right)$ and $B_{20}\left(x_{20}, y_{20}\right)$ respectively, and the analysis of the synchronization features is divided into cases depending on the initial values of the two coupled system.

Case 1: When $x_{20}-x_{10}>\delta$, as shown in Figure 4(a) with $c>0$, without the coupling term, both systems (4) and (5) are with the same slow manifold $M=\left\{(x, y) \mid y=-x+\frac{1}{3} x^{3}\right\}$, and the initial phase difference is

$T_{B_{20} A_{10}}=T_{B_{20} O}-T_{A_{10} O}$. When those two systems couple with each other, the slow manifolds of systems (4) and (5) will adjust from $M$ to

$M_{1}=\left\{(x, y) \mid y=-x+\frac{1}{3} x^{3}+c\right\}$ and $M_{2}=\left\{(x, y) \mid y=-x+\frac{1}{3} x^{3}-c\right\}$ respectively due to $x_{20}-x_{10}>\delta$. And thus, according to the geometric singular perturbation theory, the initial point $A_{10}$ will jump to $A_{11}$, and $B_{20}$ will jump to $B_{21}$, and the phase difference becomes $T_{B_{21} A_{11}}=T_{B_{21} O}-T_{A_{1} O}$ o one has

$T_{B_{21} A_{11}}<T_{B_{20} A_{10}}$. As a result, the phase difference is contracted when systems (4) and (5) adjust their slow manifolds.

Case 2: When $x_{20}-x_{10}<\delta$ and $y_{20}-y_{10}<c$, as shown in Figure 4(b) with $c>0$, the initial phase difference is $T_{B_{20} A_{10}}=T_{B_{20} O}-T_{A_{10} O}$. At the beginning when system (4) couples with system (5), both systems will not adjust their slow manifold due to $x_{20}-x_{10}<\delta$. And the slow manifold of Equation (5) will adjust from $M$ to $M_{2}$ when point $A_{10}$ jumps to $A_{11}$ due to $x_{20}-x_{11}>\delta$, and thus, at almost the same time, point $B_{20}$ will jump to $B_{21}$ following the jump of point $A_{10}$, and the phase difference becomes $T_{B_{21} A_{11}}=T_{B_{21} O}-T_{A_{11} O}$, one has $T_{B_{21} A_{11}}<T_{B_{20} A_{10}}$. As a result, the phase difference is contracted with the ratio of $I$ when systems (4) and (5) adjust their slow manifolds.

Case 3: When $x_{20}-x_{10}<\delta$ and $y_{20}-y_{10}>c$, as shown in Figure 5(a), the initial phase difference is $T_{B_{20} A_{10}}=T_{B_{20} O}-T_{A_{10} O}$. At the beginning when systems (4) and (5) couple with each other, both systems will not adjust their slow manifold due to $x_{20}-x_{10}<\delta$. And the slow manifold of systems (4) and (5) will adjust from $M$ to $M_{1}$ and $M_{2}$ respectively when point $A_{10}$ jumps to $A_{11}$ due to $x_{20}-x_{11}>\delta$, and at almost the same time, point $B_{20}$ will jump to $B_{21}$. Then point $B_{21}$ will evolve to $B_{22}$ and $A_{11}$ will evolve to $A_{12}$, and the slow manifold of systems (4) and (5) will both adjust from $M_{1}$ and $M_{2}$ to $M$ again 


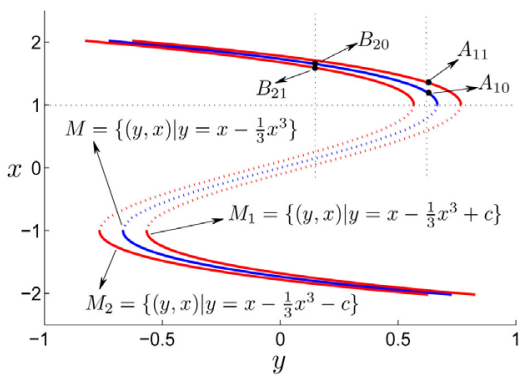

(a)

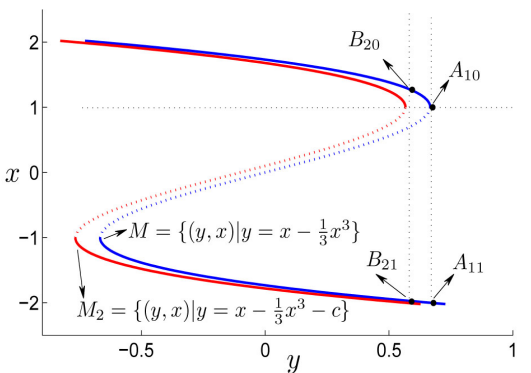

(b)

Figure 4. Equations (4) and (5) get synchronization by adjusting their slow manifolds with each other. (a) Case 1; (b) Case 2.

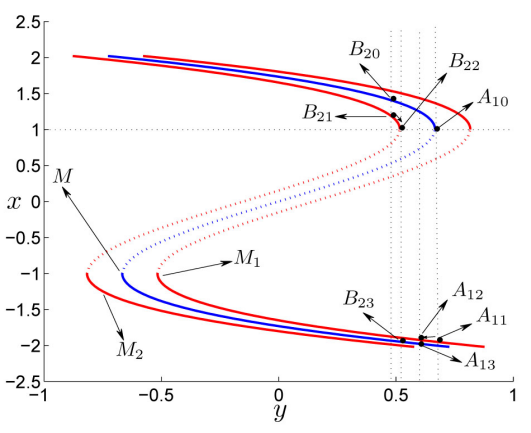

(a)

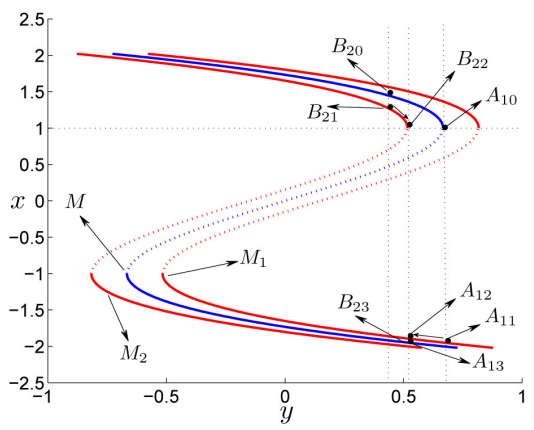

(b)

Figure 5. Equations (4) and (5) get synchronization by adjusting their slow manifolds with each other. (a) Case 3; (b) Case 3 with optimal coupling strength $c_{*}$.

when point $B_{22}$ jumps to $B_{23}$ due to $x_{23}-x_{12}<\delta$, and at almost the same time, point $A_{12}$ will jump to $A_{13}$, and one has $T_{B_{20} A_{10}}<T_{A_{13} B_{23}}<T$. As a result, the phase difference is contracted when systems (4) and (5) adjust their slow manifolds. Furthermore, as shown in Figure 5(b), there is a optimal coupling strength $c_{*}$, such that $y_{23}=y_{13}$, and systems (4) and (5) get synchronization quickly when systems (4) and (5) adjust their slow manifolds only for one time.

The analysis of cases 1 - 3 indicates the coupled slow-fast van der Pol systems can get synchronization by adjusting their slow-manifolds with each other, and this synchronization mechanism of "slow-manifold adjustment" is more complex than the mechanism of "fast threshold modulation" proposed in [25]. Moreover, one has the following results.

Results 1: When $c>0$, the coupled slow-fast van der Pol systems (2) can get synchronization through the mechanism of "slow-manifold adjustment". And there is an optimal coupling strength $c_{*}$, such that the coupled systems (2) can get synchronization quickly.

To illustrate the validity of analytical results 1 , the largest condition Lyapunov exponent of the following master stability function is calculated numerically in Figure 6(a),

$$
\left\{\begin{array}{l}
\dot{\zeta}_{1}=-\zeta_{2}+\left(1-x^{2}\right) \zeta_{1}-2 c \zeta_{1} \\
\dot{\zeta}_{2}=\varepsilon \zeta_{1} .
\end{array}\right.
$$




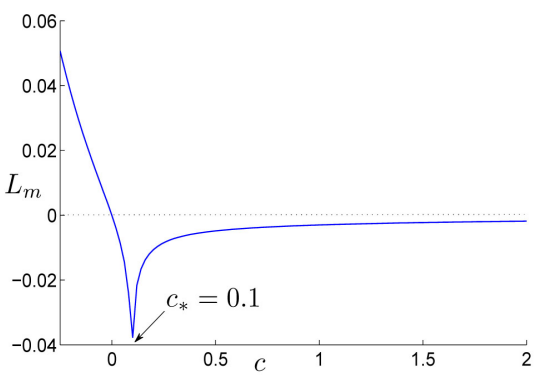

(a)

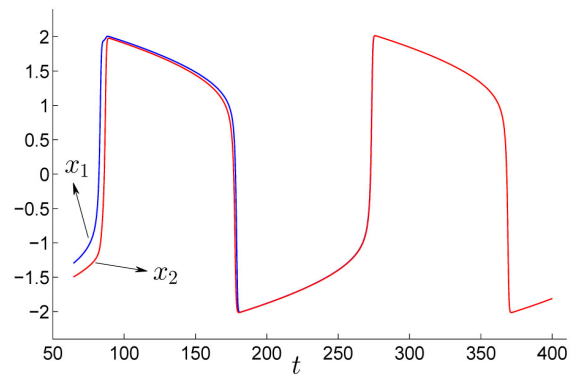

(b)

Figure 6. The largest condition Lyapunov exponent and synchtonization of coupled systems (2). (a) Largest condition Lyapunov exponent as a function of $c$, (b) Synchronization with optimal coupling strength $c_{*}$.

where $x$ is one of the variable of synchronized system (3). The coupled slow-fast van der Pol systems (2) can get synchronization when the largest condition Lyapunov exponent $L_{m}$ is negative. And there is a optimal coupling strength $c_{*}$, such that the coupled systems can get synchronization quickly, as shown in Figure 6(b). The numerical results agree with the analytical results 1.

\section{Time-Delay Effects}

Once the synchronization mechanism of coupled slow-fast van der Pol systems (2) without connection delay is clarified, time-delay effect on synchronization features can be discussed, the key step is to clarify the time-delay influence on the structure of the slow manifold due to the coupled systems (2) get synchronization by adjusting their slow-manifolds with each other, as shown in previous section.

In the synchronization manifold of coupled systems (1), the synchronized state $\left(x_{1}, y_{1}\right)=\left(x_{2}, y_{2}\right)=(x, y)$ is governed by

$$
\left\{\begin{array}{l}
\dot{x}=-y+x-\frac{1}{3} x^{3}+c(x(t-\tau)-x) \\
\dot{y}=\varepsilon x .
\end{array}\right.
$$

Let $\varepsilon \rightarrow 0$ in Equation (7), one has the fast subsystem

$$
\dot{x}=-y+x-\frac{1}{3} x^{3}+c(x(t-\tau)-x) .
$$

where $y$ is taken as a system parameter.

Geometric singular perturbation theory defines the slow manifold of Equation (7) as the set of the equilibrium points of the fast subsystem, denoted as

$$
M_{2}=M_{21} \cup M_{22} \cup M_{23}=\left\{(x, y) \mid-y+x-\frac{1}{3} x^{3}=0\right\} \text {. }
$$

Note that the set $M_{2}$ is the same as $M_{1}$, thus, the time delay has no influence on the shape of the slow manifold, as shown in Figure 7(a).

To decide time-delay influence on the stability and bifurcation of the slow manifold $M_{2}$, consider the local stability of the fast subsystem (8) around the 


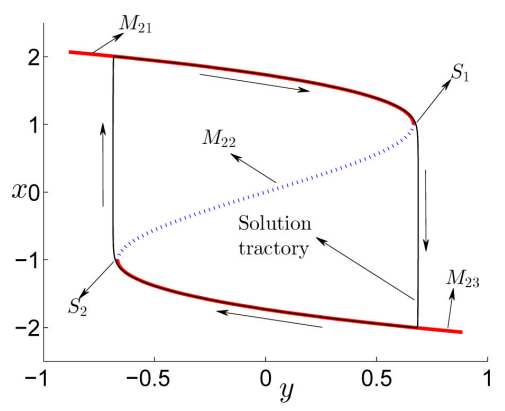

(a)

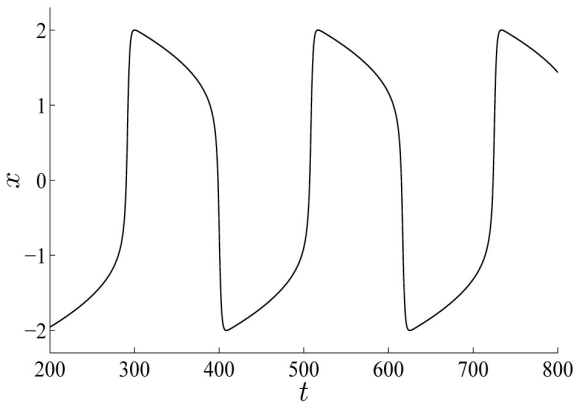

(b)

Figure 7. Slow manifold, solution trajectory and time series of Equation (7) with $\tau=O(1)>0$. (a) $M_{21}$ and $M_{23}$ are stable, and $M_{22}$ is unstable for any positive $\tau=O(1)$. (b) Equation (7) undergoes relaxation oscillation with $\tau=O(1)>0$. (a) Slow manifold and solution trajectory; (b) Time series.

equilibrium point $\left(x_{0}, y_{0}\right) \in M_{2}$, which is governed by the characteristic equation

$$
D(\lambda)=\lambda-\left(1-x_{0}^{2}\right)+c-c \mathrm{e}^{-\lambda \tau} .
$$

When $\left|x_{0}\right| \leq 1$ and $\tau=0, D(\lambda)=0$ has one nonnegative real eigenvalue $\lambda=1-x_{0}^{2} \geq 0$. According to the theory of stability switch [42], there is no stability switch as $\tau$ increases from zero to infinity. So, the second part of slow manifold $M_{22}$ is always unstable for any time delay $\tau$, as shown in Figure 7(a).

When $\left|x_{0}\right|>1$ and $\tau=0, D(\lambda)=0$ has one negative real eigenvalue $\lambda=1-x_{0}^{2}<0$, and $D(0) \neq 0$ for any $\tau$. Thus, as $\tau$ increases, stability may switch only when a couple of eigenvalues arrive in the right half plane through crossing the imaginary axis. Let $\lambda= \pm \mathrm{i} \omega \quad(\omega>0)$, and separating the real and imaginary parts of $D(\lambda)=0$ leads to

$$
\left\{\begin{array}{l}
\omega+c \sin (\omega \tau)=0, \\
\left(x_{0}^{2}-1\right)+c(1-\cos (\omega \tau))=0 .
\end{array}\right.
$$

The second equation of Equation (9) cannot hold for any $\tau$ due to $x_{0}^{2}-1>0$. Thus, there is no stability switch as $\tau$ increases from zero to infinity. So, time-delay $\tau$ cannot change the stability of the parts of the slow-manifold $M_{21}$ and $M_{23}$, and the parts of the slow manifold $M_{21}$ and $M_{23}$ are stable for any time delay $\tau$, as shown in Figure 7(a).

The analysis above indicates time delay cannot qualitatively influence the structure of the slow manifold $M_{2}$, and thus cannot qualitatively influence the dynamics of Equation (7), as shown in Figure 7(a) and Figure 7(b).

Since coupled slow-fast van der Pol systems get synchronization through the mechanism of "slow-manifold adjustment", one can conjecture that the connection delay $\tau$ cannot qualitatively affect the synchronization features of delay-coupled slow-fast van der Pol systems (1) because the time delay cannot qualitatively influence the structure of the slow manifold. To verify this conjecture numerically, the largest condition Lyapunov exponent, which governs the stability 


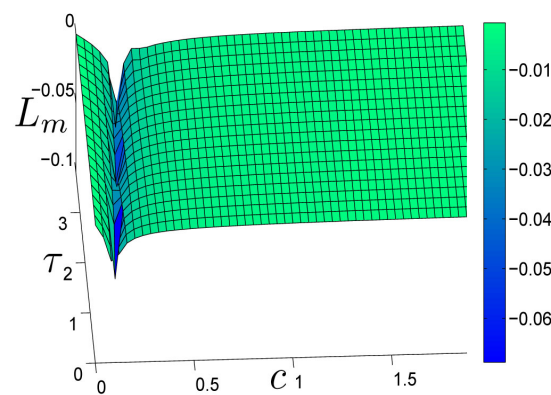

(a)

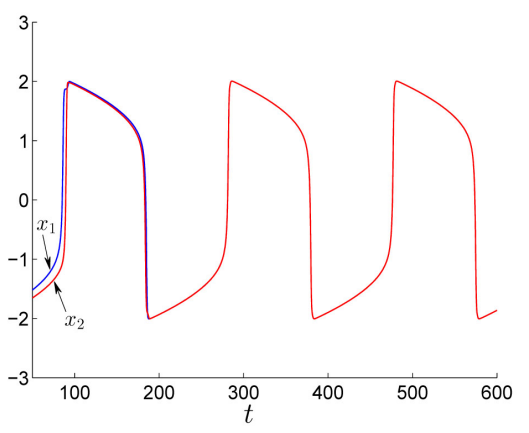

(c)

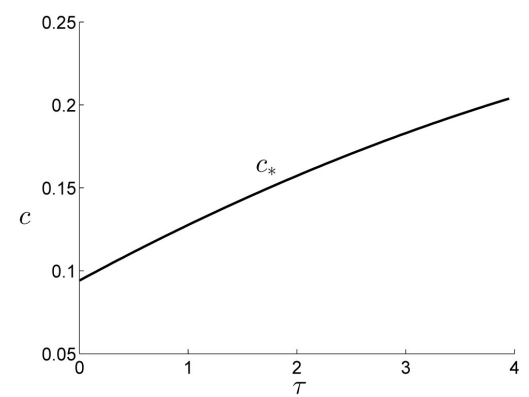

(b)

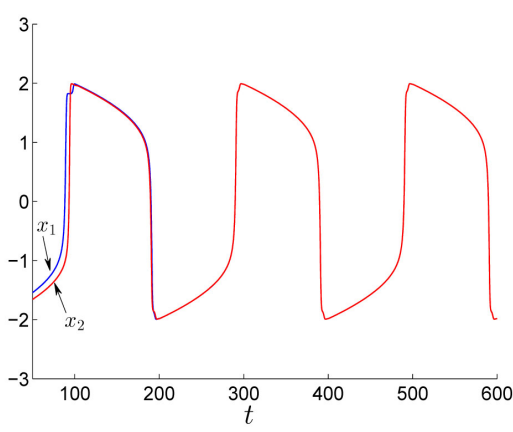

(d)

Figure 8. The largest condition Lyapunov exponents and synchronization of coupled systems (1). (a) The largest condition Lyapunov exponents $L_{m}$ as a function of $c$ and $\tau$; (b) The drift of optimal coupling strength $c_{*}$ with the increase of time delay $\tau$; (c) Synchronization of coupled systems (1) with $\tau=2$ and optimal coupling strength $c_{s}=0.16$; (d) Synchronization of coupled systems (1) with $\tau=4$ and optimal coupling strength $c_{*}=0.2$.

of synchronization manifold of coupled systems (1), is calculated from the following master stability function

$$
\left\{\begin{array}{l}
\dot{\zeta}_{1}=-\zeta_{2}+\left(1-x^{2}\right) \zeta_{1}-c\left(\zeta_{1}(t-\tau)+\zeta_{1}\right), \\
\dot{\zeta}_{2}=\varepsilon \zeta_{1}
\end{array}\right.
$$

where $x$ is the variable of Equation (7).

The largest condition Lyapunov exponents as a function of $c$ and $\tau$ are illustrated in Figure 8(a), which verifies the analytical conjecture that the time delay $\tau$ cannot qualitatively affect the synchronization mechanism of "slow-manifold adjustment", and furthermore, it is shown that the time delay can result to the drift of the optimal coupling strength $c_{*}$, as shown in Figures 8(b)-(d).

Summarize the analytical and numerical results, one has:

Results 2: The time delay $\tau$ cannot qualitatively affect the synchronization mechanism of "slow-manifold adjustment", however, it can result to the drift of the optimal coupling strength $c_{*}$.

\section{Conclusion}

Coupled slow-fast van der Pol systems can describe the dynamics of various real systems in physics, electronic, and biology. And connection delay should be 
considered due to limit of information transmission and processing speed. It is well known time-delay that is often a negative factor to the stability of synchronization, and deteriorates the synchronizability of coupled systems with uniform timescale. The studies in this paper indicate even large time-delay cannot destroy the synchronization mechanism of "slow-manifold adjustment" of coupled slow-fast systems. The finding is helpful to understand the universal synchronization in real systems with long connection delay.

\section{Funding}

This paper was supported by NSF of China under Grants 11872197, 11202187, JXNSF and JXECF of China under Grant 20192BAB202002 and DB201407058 respectively.

\section{Conflicts of Interest}

The authors declare no conflicts of interest regarding the publication of this paper.

\section{References}

[1] Eckhaus, W. (1983) Relaxation Oscillations Including a Standard Chase on French Ducks. In: Verhulst, F., Ed., Asymptotic Analysis, II, volume 985 of Lecture Notes in Math, Springer-Verlag, Berlin, 449-494. https://doi.org/10.1007/BFb0062381

[2] Izhikevich, E.M. (2000) Neural Excitability, Spiking and Bursting. International Journal of Bifurcation and Chaos, 10, 1171-1266. https://doi.org/10.1142/S0218127400000840

[3] van der Pol, B. (1926) On "Relaxation-Oscillations". The London, Edinburgh, and Dublin Philosophical Magazine and Journal of Science, 2, 978-992. https://doi.org/10.1080/14786442608564127

[4] Diener, F. and Diener, M. (1983) Sept Formules Relatives Aux Canards. Les Comptes Rendus de I Acadmie des Sciences, 297, 577-580.

[5] Dumortier, F. and Roussarie, R. (1996) Canard Cycles and Center Manifolds. Memoirs of the American Mathematical Society, 121, 577. https://doi.org/10.1090/memo/0577

[6] Krupa, M. and Szmolyan, P. (2001) Extending Slow Manifolds near Transcritical and Pitchfork Singularities. Nonlinearity, 14, 1473-1491. https://doi.org/10.1088/0951-7715/14/6/304

[7] Liu, W., Xiao, D. and Yi, Y. (2003) Relaxation Oscillations in a Class of Predator Prey Systems. Journal of Differential Equations, 188, 306-331. https://doi.org/10.1016/S0022-0396(02)00076-1

[8] Chumakov, G. and Chumakova, N. (2003) Relaxation Oscillations in a Kinetic Model of Catalytic Hydrogen Oxidation Involving a Chase on Canards. Chemical Engineering Journal, 91, 151-158. https://doi.org/10.1016/S1385-8947(02)00148-1

[9] FitzHugh, R. (1961) Impulses and Physiological States in Models of Nerve Membrane. Biophysical Journal, 1, 445-466. https://doi.org/10.1016/S0006-3495(61)86902-6

[10] Morris, C. and Lecar, H. (1981) Voltage Oscillations in the Barnacle Giant Muscle Fiber. Biophysical Journal, 35, 193-213. 
https://doi.org/10.1016/S0006-3495(81)84782-0

[11] Li, X., Jefferys, J.G.R., Fox, J. and Yao, X. (2008) Neuronal Population Oscillations of Rat Hippocampus during Epileptic Seizures. Neural Networks, 21, 1105-1111. https://doi.org/10.1016/j.neunet.2008.06.002

[12] Zheng, Y.G. and Wang, Z.H. (2012) Relaxation Oscillation and Attractive Basins of a Two-Neuron Hopfield Network with Slow and Fast Variables. Nonlinear Dynamics, 70, 1231-1240. https://doi.org/10.1007/s11071-012-0527-7

[13] Tikhonov, A.N. (1952) Systems of Differential Equations Containing a Small Parameter Multiplying the Derivative. Mathematical Sbornik, 31, 575-586.

[14] Fenichel, N. (1974) Asymptotic Stability with Rate Conditions. Indiana University Mathematics Journal, 23, 1109-1137. https://doi.org/10.1512/iumj.1974.23.23090

[15] Fenichel, N. (1979) Geometric Singular Perturbations Theory for Ordinary Differential Equations. Journal of Differential Equations, 31, 53-98. https://doi.org/10.1016/0022-0396(79)90152-9

[16] Jones, C. (1994) Geometric Singular Perturbation Theory. In: Johnson, R., Ed., Dynamical Systems, Springer-Verlag, Berlin, 44-118. https://doi.org/10.1007/BFb0095239

[17] Huygens, C. (1673) Horologium oscillatorium. Apud F. Muguet, Parisiis.

[18] Rosenblum, M., Pikovsky, A. and Kurths, J. (1997) From Phase to Lag Synchronization in Coupled Chaotic Oscillators. Physical Review Letters, 78, 4193-4196. https://doi.org/10.1103/PhysRevLett.78.4193

[19] Pikovsky, A., Rosenblum, M. and Kurths, J. (2001) Synchronization: A Universal Concept in Nonlinear Sciences. Cambridge University Press, New York. https://doi.org/10.1017/CBO9780511755743

[20] Wong, W.K., Zhen, B., Xu, J. and Wang, Z. (2012) An Analytic Criterion for Generalized Synchronization in Unidirectionally Coupled Systems Based on the Auxiliary System Approach. Chaos, 22, 033146. https://doi.org/10.1063/1.4748862

[21] Pecora, L. and Carroll, T. (1990) Synchronization in Chaotic Systems. Physical Review Letters, 64, 821-824. https://doi.org/10.1103/PhysRevLett.64.821

[22] Kim, C.-M., Rim, S., Kye, W.-H., Ryu, J.-W. and Park, Y.-J. (2003) Anti-Synchronization of Chaotic Oscillators. Physics Letters A, 320, 39-46.

https://doi.org/10.1016/j.physleta.2003.10.051

[23] Pecora, L., Carroll, T., Johnson, G. and Mar, D. (1997) Fundamentals of Synchronization in Chaotic Systems, Concepts, and Applications. Chaos, 7, 520-543. https://doi.org/10.1063/1.166278

[24] Pecora, L. and Carroll, T. (1998) Master Stability Functions for Synchronized Coupled System. Physical Review Letters, 80, 2109-2112. https://doi.org/10.1103/PhysRevLett.80.2109

[25] Somers, D. and Kopell, N. (1993) Rapid Synchronization through Fast Threshold Modulation. Biological Cybernetics, 68, 393-407.

https://doi.org/10.1007/BF00198772

[26] Izhikevich, E.M. (2000) Phase Equations for Relaxation Oscillators. SIAM Journal on Applied Mathematics, 60, 1789-1805. https://doi.org/10.1137/S0036139999351001

[27] Campbell, S.R., Wang, D.L. and Jayaprakash, C. (2004) Synchronization Rates in Classes of Relaxation Oscillators. IEEE Transactions on Neural Networks, 15, 1027-1038. https://doi.org/10.1109/TNN.2004.833134

[28] Varkonyi, P.L. and Holmes, P. (2008) On Synchronization and Traveling Waves in 
Chains of Relaxation Oscillators with an Application to Lamprey CPG. SIAM Journal on Applied Dynamical Systems, 7, 766-794. https://doi.org/10.1137/070710329

[29] Singh, R. and Sinha, S. (2013) Spatiotemporal Order, Disorder, and Propagating Defects in Homogeneous System of Relaxation Oscillators. Physical Review E, 87, 012907. https://doi.org/10.1103/PhysRevE.87.012907

[30] Reddy, K.R., Sen, A. and Johnston, G. (1998) Time Delay Induced Death in Coupled Limit Cycle Oscillators. Physical Review Letters, 80, 5109-5112. https://doi.org/10.1103/PhysRevLett.80.5109

[31] Nakamura, Y., Tominaga, F. and Munakata, T. (1994) Clustering Behavior of Time-Delayed Nearest-Neighbor Coupled Oscillators. Physical Review E, 49, 48494856. https://doi.org/10.1103/PhysRevE.49.4849

[32] Dhamala, M., Jirsa, V.K. and Ding, M. (2004) Enhancement of Neural Synchrony by Time Delay. Physical Review Letters, 92, 074104. https://doi.org/10.1103/PhysRevLett.92.074104

[33] Echi, N. and Benabdallah, A. (2017) Delay-Dependent Stabilization of a Class of Time-Delay Nonlinear Systems: LMI Approach. Advances in Difference Equations, 2017, Article No. 271. https://doi.org/10.1186/s13662-017-1335-7

[34] Mukdasai, K. and Kaewbanjak, N. (2019) On Delay-Interval-Dependent Robust Stability of Lpddiscrete-Time System with Mixed Time-Varying Delays and Nonlinear Uncertainties. Advances in Difference Equations, 2019, Article No. 242. https://doi.org/10.1186/s13662-019-2179-0

[35] Atay, F., Jost, J. and Wende, A. (2004) Delays, Connection Topology, and Synchronization of Coupled Chaotic Maps. Physical Review Letters, 92, 144101. https://doi.org/10.1103/PhysRevLett.92.144101

[36] Zheng, Y. and Wang, Z. (2012) Time-Delay Effect on the Bursting of the Synchronized State of Coupled Hindmarsh-Rose Neurons. Chaos, 22, 043127. https://doi.org/10.1063/1.4768664

[37] Wang, Q., Perc, M., Duan, Z. and Chen, G. (2009) Synchronization Transitions on Scale-Free Neuronal Networks Due to Finite Information Transmission Delays. Physical Review E, 80, 026206. https://doi.org/10.1103/PhysRevE.80.026206

[38] Tang, J., Ma, J., Yi, M., Xia, H. and Yang, X. (2011) Delay and Diversity-Induced Synchronization Transitions in a Small-World Neuronal Network. Physical Review E, 83, 046207. https://doi.org/10.1103/PhysRevE.83.046207

[39] Wang, Q. and Lu, Q. (2005) Time Delay-Enchance Synchronization and Regularization in Two Coupled Chaotic Neurons. Chinese Physics Letters, 22, 543-546. https://doi.org/10.1088/0256-307X/22/3/007

[40] Campbell, S.R. and Wang, D.L. (1998) Relaxation Oscillators with Time Delay Coupling. Physica D, 111, 151-178. https://doi.org/10.1016/S0167-2789(97)80010-3

[41] Fox, J.J., Jayaprakash, C., Wang, D.L. and Campbell, S.R. (2001) Synchronization in Relaxation Oscillator Networks with Conduction Delays. Neural Computation, 13, 1003-1021. https://doi.org/10.1162/08997660151134307

[42] Wang, Z.H. and Hu, H.Y. (2000) Stability Switches of Time-Delayed Dynamic Systems with Unknown Parameters. Journal of Sound and Vibration, 233, 215-233. https://doi.org/10.1006/jsvi.1999.2817 